\title{
Spin-diffusion effect in the ferromagnet-metal bilayer
}

\author{
Sui-Pin Chen*, Ching-Ray Chang \\ Department of Physics, Center of Nanostorage Research, National Taiwan University, 10617 Taipei, Taiwan
}

\begin{abstract}
Spin accumulation in a ferromagnet-metal bilayer is investigated by means of the Boltzmann transport equation. We consider the spin-dependent diffusive scatterings from different mean free paths between the ferromagnet and the metal, and find that the resultant spin-diffusion length depends both on the interfacial properties and on the mean free paths, and that it usually is less than the metal mean free path.
\end{abstract}

(C) 2003 Elsevier B.V. All rights reserved.

PACS: $75.40 . \mathrm{Gb} ; 72.25 . \mathrm{Mk} ; 73.40$

Keywords: Spin-dependent diffusive scattering; Spin accumulation; Chemical potential

Spin accumulation occurs when a current flows across the interfaces of magnetic multilayers [1-3]. P.C. van Son et al. used a phenomenological diffusion equation [1], derived by Valet and Fert from the Boltzmann transport equation [2], to specify the variation of the chemical potential with the so-called spin-diffusion length on the scale of a few micrometers [1-3]. However, the experimentally observed spin-diffusion length seems to be only a few nanometers, and the tunneling magnetoresistance ratio of the ferromagnet-metal-insulator-ferromagnet junction decays thus rapidly with increasing metal thickness [4-7]. In this paper, we consider the spin-dependent diffusive scatterings from different mean free paths in a ferromagnet-metal bilayer, and find the resultant spin-diffusion length to be well below the metal mean free path. Moreover, it can be even zero in particular cases.

The spin-dependent electron distribution with boundary effects and in the presence of a uniform electric field $\mathbf{E}$ is given by the formula

$f_{\mathbf{k}}^{\sigma}(\varepsilon, \mathbf{r})=\frac{1}{\exp \left[\beta\left(\varepsilon-\mu_{\mathbf{k}}^{\sigma}(\mathbf{r})\right)\right]+1}$,

where $\varepsilon$ is the electron energy, and $\mathbf{k}$ is its wave vector at the position $\mathbf{r}$. The spin-dependent chemical

\footnotetext{
*Corresponding author. Tel.: + 886-2-3366-5188; fax: + 8862-2363-9984.

E-mail address: d89222002@ms89.ntu.edu.tw (S.-P. Chen).
}

potential is

$\mu_{\mathbf{k}}^{\sigma}(\mathbf{r})=\mu^{0}+\sigma h-\lambda^{\sigma} \mathbf{e}_{\mathbf{k}} \cdot e \mathbf{E}+\Delta \mu_{\mathbf{k}}^{\sigma}(\mathbf{r})$,

where $\mu^{0}$ is the spin-independent chemical potential, the spinor $\sigma$ is $+1(-1,0)$ for the spin-up (spin-down, spinindependent) state, $h$ is the molecular field of the ferromagnet, $\sigma h$ is its internal exchange energy, $\lambda^{\sigma}$ is the electron mean free path, $-e$ is the electron charge, and $\mathbf{e}_{\mathbf{k}}$ is a unit vector in the transport direction. In Eq. (2) the anisotropic chemical potential change $-\lambda^{\sigma} \mathbf{e}_{\mathbf{k}} \cdot e \mathbf{E}$ is due to the applied electric field $\mathbf{E}$, and the boundary chemical potential change $\Delta \mu_{\mathbf{k}}^{\sigma}(\mathbf{r})$ encompasses all possible induced boundary effects. The electron distribution $f_{\mathbf{k}}^{\sigma}(\varepsilon, \mathbf{r})$ is required to satisfy the Boltzmann transport equation in the relaxation time approximation,

$\frac{\mathrm{d} f_{\mathbf{k}}^{\sigma}(\varepsilon, \mathbf{r})}{\mathrm{d} t}=-\frac{f_{\mathbf{k}}^{\sigma}(\varepsilon, \mathbf{r})-f_{\mathbf{k}}^{\sigma, 0}(\varepsilon)}{\tau^{\sigma}}$,

where $f_{\mathbf{k}}^{\sigma, 0}(\varepsilon)$ is the Fermi-Dirac equilibrium distribution and $\tau^{\sigma}$ is the relaxation time. The boundary chemical potential change $\Delta \mu_{\mathbf{k}}^{\sigma}(\mathbf{r})$ therefore is valid under

$\mathbf{e}_{\mathbf{k}} \cdot \nabla_{\mathbf{r}}\left[\Delta \mu_{\mathbf{k}}^{\sigma}(\mathbf{r})\right]=-\frac{\Delta \mu_{\mathbf{k}}^{\sigma}(\mathbf{r})}{\lambda^{\sigma}}$,

where the mean free path is $\lambda^{\sigma}=v_{\mathrm{F}}^{\sigma} \tau^{\sigma}$ and the Fermi velocity is $v_{\mathrm{F}}^{\sigma}$. In the ferromagnet-metal bilayer, the 
electric field $\mathbf{E}$ is applied parallel to the $z$-axis, which is perpendicular to the ferromagnet-metal interface. The film thickness of the left ferromagnet and of the right metal are designated as $a$ and $b$, respectively. Accordingly, the electron distribution $f_{\mathbf{k}}^{\sigma}(\varepsilon, \mathbf{r})$ and the boundary chemical potential change $\Delta \mu_{\mathbf{k}}^{\sigma}(\mathbf{r})$ follow from Eqs. (1)-(3):

$$
\begin{aligned}
f_{\mathbf{k}}^{\sigma}(\varepsilon, \mathbf{r})= & f_{\mathbf{k}}^{\sigma, 0}(\varepsilon)+\frac{\partial f_{\mathbf{k}}^{\sigma, 0}(\varepsilon)}{\partial \varepsilon} \\
& \times\left[e \lambda^{\sigma} E \cos \theta-G_{\mathbf{k}}^{\sigma} \exp \left[\frac{-z}{\lambda^{\sigma} \cos \theta}\right]\right],
\end{aligned}
$$

$\Delta \mu_{\mathbf{k}}^{\sigma}(\mathbf{r})=G_{\mathbf{k}}^{\sigma} \exp \left[\frac{-z}{\lambda^{\sigma} \cos \theta}\right]$

where $\cos \theta=\mathbf{e}_{\mathbf{k}} \cdot \mathbf{e}_{\mathbf{z}}$. The undetermined coefficient $G_{\mathbf{k}}^{\sigma}$ depends on the boundary conditions, including the diffusion parameter $D$, the non-diffusive transmission parameter $T$, and the non-diffusive reflection parameter $R$. In the following, we denote all physical variables by the subscripts F, M, and F-M in order to indicate the related outer surfaces and the interface of the ferromagnet-metal bilayer.

To study the spin accumulation within the metal, which results from the injected spin-dependent electrons of the ferromagnet, we assume the boundary conditions as follows; $D_{\mathrm{F}}=0, T_{\mathrm{F}}=0$, and $R_{\mathrm{F}}=1$ for the source at $z=-a, D_{\mathrm{M}}=0, T_{\mathrm{M}}=1$, and $R_{\mathrm{M}}=0$ for the drain at $z=b$, and $D_{\mathrm{F}-\mathrm{M}}=D, T_{\mathrm{F}-\mathrm{M}}=1-D$, and $R_{\mathrm{F}-\mathrm{M}}=0$ for the ferromagnet-metal interface at $z=0$. As the ferromagnet is a good spin-polarized source for the metal drain, it is reasonable to approximate $2 a \gg \lambda_{\mathrm{F}}^{+}$, $2 a \gg \lambda_{\mathrm{F}}^{-}$, and $a>b$. After some algebra, the difference ratio between the spin-dependent chemical potentials within the metal can be derived as

$$
\begin{aligned}
\frac{\mu_{\mathbf{k}, \mathrm{M}}^{+}(z)-\mu_{\mathbf{k}, \mathrm{M}}^{-}(z)}{\mu_{\mathrm{M}}^{0}}= & \frac{\left(e E \lambda_{\mathrm{M}} \cos \theta\right)}{\mu_{\mathrm{M}}^{0}}\left(D_{\mathrm{F}-\mathrm{M}}^{+}-D_{\mathrm{F}-\mathrm{M}}^{-}\right) \\
& \times \exp \left[\frac{-z}{\lambda_{\mathrm{M}} \cos \theta}\right],
\end{aligned}
$$

where $\lambda_{\mathrm{M}}^{+}=\lambda_{\mathrm{M}}^{-}=\lambda_{\mathrm{M}}$ and the spin-dependent diffusive scattering at the ferromagnet-metal interface is given by [8]

$D_{\mathrm{F}-\mathrm{M}}^{\sigma}=\left[1-\frac{\lambda_{\mathrm{F}}^{\sigma}}{\lambda_{\mathrm{M}}^{\sigma}}(1-D)\right]$.

Eqs. (5a) and (5b) show that the asymmetry of the different spin-dependent mean free paths between the ferromagnet and the metal, $\lambda_{\mathrm{F}}^{+} / \lambda_{\mathrm{M}}^{+} \neq \lambda_{\mathrm{F}}^{-} / \lambda_{\mathrm{M}}^{-}$, causes the asymmetry of the different spin-dependent diffusive scatterings, $D_{\mathrm{F}-\mathrm{M}}^{+} \neq D_{\mathrm{F}-\mathrm{M}}^{-}$. This is one reason for the spin accumulation with $\mu_{\mathbf{k}, \mathbf{M}}^{+}(z) \neq \mu_{\mathbf{k}, \mathbf{M}}^{-}(z)$ in the ferro- magnet-metal bilayer. It is clear from Eqs. (5a) and (5b) that the interfacial diffusive scattering, i.e., $D$, and the incident angle $\theta$ strongly effect the spin accumulation injected from the ferromagnet into the metal. Moreover, a larger spin accumulation occurs at higher values of the ratio $\left(\lambda_{\mathrm{F}}^{+}-\lambda_{\mathrm{F}}^{-}\right) / \lambda_{\mathrm{M}}$. The metal spin-diffusion length $\Lambda$ is defined to specify the existence of the spin accumulation, which is assumed as a distance from the ferromagnetmetal interface with $\mu_{\mathrm{M}}^{0} \exp [-1]$. In a good case, with $\left(e E \lambda_{\mathrm{M}}\right) / \mu_{\mathrm{M}}^{0}=1,\left(\lambda_{\mathrm{F}}^{+}-\lambda_{\mathrm{F}}^{-}\right) / \lambda_{\mathrm{M}}=1, \theta=0$, and $D=0$, the spin-diffusion length $\Lambda$ is the mean free path $\lambda_{\mathrm{M}}$. In contrast, no spin accumulation occurs for either $D=1$ or $\theta=\pi / 2$, where $\Lambda=0$.

In summary, the spin-diffusion length within the metal part of the ferromagnet-metal bilayer is due to the fact that the spin-dependent electrons injected from the ferromagnet undergo different spin-dependent diffusive scatterings, i.e., $D_{\mathrm{F}-\mathrm{M}}^{+}$and $D_{\mathrm{F}-\mathrm{M}}^{-}$, near the ferromagnetmetal interface. The spin-diffusion length is comparable to the mean free path in metal only under good conditions. If the interfacial diffusive scatterings or the smaller asymmetry of the spin-dependent diffusive scatterings take place, the spin-diffusion length becomes much less than the mean free path in metal, and can even down to zero in particular cases. This is a possible reason for the reported experimental small spin-diffusion length [4-7].

This research was supported by National Science Council of Taiwan under Grants NSC-91-2120-M-002003 and Ministry of Economic Affairs of Taiwan under Grants 91-EC-17-A-08-S1-0006.

\section{References}

[1] P.C. van Son, H. van Kempen, P. Wyder, Phys. Rev. Lett. 58 (1985) 2271.

[2] T. Valet, A. Fert, Phys. Rev. B 48 (1993) 7099.

[3] M. Johnson, R.H. Silsbee, Phys. Rev. Lett. 55 (1985) 1790.

[4] S. Yuasa, T. Nagahama, Y. Suzuki, Science 297 (2002) 234.

[5] P. LeClair, B. Hoex, H. Wieldraaijer, J.K. Kohlhepp, H.J.M. Swagten, W.J.M. de Jonge, Phys. Rev. B 64 (2001) 100406.

[6] J.S. Moodera, J. Nowak, L.R. Kinder, P.M. Tedrow, R.J.M. van de Veerdonk, B.A. Smits, M. Van Kampen, H.J.M. Swagten, W.J.M. de Jonge, Phys. Rev. Lett. 83 (1999) 3029.

[7] P. LeClair, H.J.M. Swagten, J.K. Kohlhepp, R.J.M. van de Veerdonk, W.J.M. de Jonge, Phys. Rev. Lett. 84 (2000) 2933.

[8] Ching-Ray Chang, Sui-Pin Chen, unpublished. 